\title{
Increased Serum Uric Acid Levels Are Correlated with Decreased Left Atrial Appendage Peak Flow Velocity in Patients with Atrial Fibrillation
}

\author{
Murat Celik $^{a} \quad$ Emre Yalcinkaya $^{\mathrm{b}} \quad$ Uygar Cagdas Yuksel $^{\mathrm{a}}$ Yalcin Gokoglan $^{\mathrm{a}}$ \\ Baris Buganc Hasan Kutsi Kabul ${ }^{a}$ Cem Barcin ${ }^{a}$

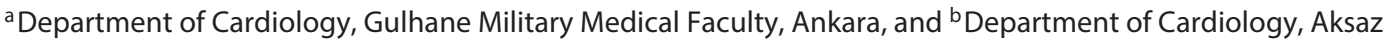 \\ Military Hospital, Aksaz/Marmaris/Mugla, Turkey; ${ }^{\mathrm{C}}$ Girne Military Hospital, Girne, Cyprus
}

\section{Key Words}

Atrial fibrillation · Left atrial appendage peak flow velocity · Serum uric acid level

\begin{abstract}
Objective: We aimed to examine the relationship between serum uric acid levels and left atrial appendage (LAA) peak flow velocity, an indicator of the mechanical functions of the LAA, and atrial fibrillation (AF). Subjects and Methods: Transesophageal echocardiography was performed before cardioversion in 153 patients with AF. The patients were categorized into 2 groups based on their LAA blood flow velocity. Group 1 included 87 patients with a low LAA flow velocity $(<35 \mathrm{~cm} / \mathrm{s})$, and group 2 comprised 66 patients with a normal LAA flow velocity $(\geq 35 \mathrm{~cm} / \mathrm{s})$. The $x^{2}$ and Student's $t$ tests were used to compare categorical and quantitative data between the groups. Linear regression analyses were performed to demonstrate the independent association between serum uric acid levels and LAA peak flow velocity. $\boldsymbol{R} \boldsymbol{e}$ sults: The LAA blood flow velocity was $24.62 \pm 5.90 \mathrm{~cm} / \mathrm{s}$ in group 1 and $49.28 \pm 13.72 \mathrm{~cm} / \mathrm{s}$ in group 2 , respectively $(\mathrm{p}<$ $0.001)$. The serum uric acid levels were $6.88 \pm 1.85 \mathrm{mg} / \mathrm{dl}$ in group 1 and $5.97 \pm 1.51 \mathrm{mg} / \mathrm{dl}$ in group 2, and the difference was statistically significant $(p=0.001)$. There was a negative correlation between serum uric acid levels and LAA blood
\end{abstract}

flow velocity ( $r=-0.216, p=0.007$ ). Multivariate regression analysis showed that serum uric acid levels, age and gender differences were significant predictors of the LAA peak flow velocity. Conclusions: High serum uric acid levels were associated with a low contractile function of the LAA and could provide additional prognostic information on future thromboembolic events in patients with AF.

(c) 2015 S. Karger AG, Basel

\section{Introduction}

The risk of thromboembolism is affected by numerous factors and shows considerable variability among patients with atrial fibrillation (AF) [1]. Coexisting clinical, echocardiographic and biochemical factors, which increase the risk of thromboembolic events in patients with AF, have been identified $[1,2]$.

Serum uric acid is the end product of purine metabolism catalyzed by xanthine oxidoreductase (XO) [1]. Elevated serum uric acid levels have been reported as an independent risk factor of cardiovascular morbidity and mortality, especially in patients with heart failure and AF $[2,3]$. The XO enzyme activity has been reported, and, hence, in the left atrium (LA), elevated uric acid levels may be a sign of oxidative damage and inflamma-

\begin{tabular}{ll}
\hline KARGER 125/s & $\begin{array}{l}\text { ○ 2015 S. Karger AG, Basel } \\
1011-7571 / 15 / 0243-0263 \$ 39.50 / 0 \quad \text { Karger }\end{array}$ \\
E-Mail karger@karger.com & $\begin{array}{l}\text { Thisis an Open Access article licensed under the terms of the } \\
\text { Creative Commons Attribution-NonCommercial 3.0 Un- } \\
\text { ported license (CC BY-NC) (www.karger.com/OA-license), } \\
\text { applicable to the online version of the article only. Distribu- } \\
\text { tion permitted for non-commercial purposes only. }\end{array}$
\end{tabular}

Emre Yalcinkaya, MD

Department of Cardiology

Aksaz Military Hospital

TR-48750 Aksaz/Marmaris/Mugla (Turkey)

E-Mail dremreyalcinkaya@gmail.com 
tion, which may play a role in the pathogenesis of $\mathrm{AF}$ [4-6].

The left atrial appendage (LAA) is a blind-ended pouch, which is embryologically distinct from the main body of the LA. The contractile function of the LAA has been shown to provide a significant contribution to the overall compliance and reservoir function of the LA [7]. AF can lead to structural remodeling of the LA, causing reduced contractile function of the LA and/or the LAA. Most of the clinical interest in LAA peak flow velocity arises from its relationship with thromboembolism [5]. Both surgical and autopsy studies have shown that thromboembolic complications develop during $\mathrm{AF}$ and are mostly due to the emboli or thrombi formed during blood stasis in the LAA [7]. Thus, the contractile function of the LAA and the degree of blood stasis within the LAA have been regarded as substantial determinants of thromboembolic risk in patients with AF. Although much is known about the role of serum uric acid in atrial remodeling, less is known about the potential pathogenetic role of uric acid in maintaining the function of the LAA.

Therefore, in this study, the association between serum uric acid levels and LAA peak flow velocity was assessed using transesophageal echocardiography (TEE) in patients with AF, which may provide additional information about the pathogenesis of thromboembolic events in this patient group.

\section{Subjects and Methods}

\section{Patient Selection}

This was a retrospective study, and patients with AF, who were admitted to the Department of Cardiology and in whom TEE was performed before cardioversion, were retrospectively screened via the hospital's computerized patient database. Exclusion criteria were: thyroid dysfunction, end-stage heart failure, hepatic failure, recent acute myocardial infarction, malignancies, blood dyscrasias, autoimmune or inflammatory diseases and the use of drugs, which may potentially interfere with the uric acid metabolism, such as allopurinol, diuretics, anti-inflammatories and antioxidants.

A total of 231 patients were evaluated, and 56 patients were excluded from the study. Twenty-two patients who did not have serum uric acid levels on file were also excluded from the study. Hence, 153 patients were included in the study. After the assessment of baseline demographic and clinical characteristics, patients were divided into 2 subgroups according to their LAA peak flow velocity. Patients with a low LAA peak flow velocity $(<35 \mathrm{~cm} / \mathrm{s})$ were identified as group 1 , and patients with a normal LAA peak flow velocity $(\geq 35 \mathrm{~cm} / \mathrm{s})$ were identified as group 2 .

\section{Transesophageal Echocardiography}

All the patients included in the study had undergone TEE examination for left atrial thrombus investigation. The TEE record- ings were re-examined offline irrespective of the previous results by 2 echocardiography specialists (M.C. and Y.G.) who were blinded to the data of the patients. The TEE examinations were performed after an overnight fasting period in the left lateral decubitus position with the application of $10 \%$ lidocaine spray to the hypopharynx (Philips iE 336.0 ultrasound system and Live 3D TEE probe X7-2t; Philips Medical Systems, Andover, Mass., USA). LAA blood flow velocity was measured by placing the pulsed-wave Doppler sample volume at the orifice of the LAA, and the peak outflow velocity signals were averaged over a minimum of six cardiac cycles. The presence of a thrombus and spontaneous echocardiographic contrast (SEC) was also evaluated. All TEE findings and the transthoracic echocardiography findings that have been made before the TEE procedure were compared between the 2 groups.

\section{Laboratory Measurements}

Blood samples for standard biochemical tests were obtained in the early morning hours 1 or 2 days prior to TEE examination. In all patients, the serum uric acid level, fasting plasma glucose, serum urea, serum creatinine, total serum cholesterol, triglyceride, highdensity lipoprotein and low-density lipoprotein cholesterol were measured with an Olympus AU2700 autoanalyzer using its own kits (Olympus Diagnostics, Hamburg, Germany) in the central laboratory of our institute. The estimated glomerular filtration rate (eGFR) was measured using the 4-variable modifications of diet in renal disease equation.

\section{Statistical Analysis}

Continuous variables were expressed as the mean \pm standard deviation and categorical variables as percentages. Continuous variables were also checked for the normal distribution assumption using the Kolmogorov-Smirnov statistics. The $\chi^{2}$ and Student's $t$ tests were used to compare categorical and quantitative data between the 2 groups, respectively. Pearson's and Spearman's coefficients were used to perform an analysis of correlation. A univariate linear regression analysis was performed to demonstrate the independent association between serum uric acid levels and LAA peak flow velocity. To determine the independent influence of serum uric acid levels on LAA blood flow velocity, multivariate linear regression analysis was performed, using the backward method based on various independent variables, which likely affect the LAA peak flow velocity. A p value $<0.05$ was considered statistically significant. All statistical analyses and calculations were conducted using the SPSS 20.0 Statistical Package Program for Windows (SPSS, Chicago, Ill., USA).

The Institutional Ethics Committee approved this study protocol, and the study was conducted according to the regulations of the Declaration of Helsinki.

\section{Results}

Of the 153 patients, 91 were men (59.5\%) and 62 were women $(40.5 \%)$, with a mean age of $65.92 \pm 12.92$ years. Group 1 consisted of 87 patients [ 43 men (49.4\%) and 44 women $(50.6 \%)$, with a mean age of $68.3 \pm 9.9$ years], and group 2 consisted of 66 patients [ 48 men (72.7\%) and 18 women $(27.3 \%)$, with a mean age of $62.8 \pm 15.5$ years]. 
Table 1. Demographics, clinical and biochemical data of AF patients categorized according to LAA blood flow velocity

\begin{tabular}{lccc}
\hline & Group 1 $(\mathrm{n}=87)$ & Group 2 (n=66) & $\mathrm{p}$ \\
\hline Age, years & $68.3 \pm 9.9$ & $62.8 \pm 15.5$ & 0.010 \\
Females & $44(50.6)$ & $18(27.3)$ & 0.003 \\
Coronary artery disease & $31(35.6)$ & $18(27.3)$ & 0.178 \\
Hypertension & $46(52.9)$ & $31(47.0)$ & 0.288 \\
Hyperlipidemia & $18(20.7)$ & $10(15.2)$ & 0.254 \\
Diabetes mellitus & $20(23.0)$ & $14(21.2)$ & 0.476 \\
Fasting glucose, $\mathrm{mg} / \mathrm{dl}$ & $110.6 \pm 35.1$ & $119.5 \pm 50.3$ & 0.202 \\
GFR, ml/min/1.73 m ${ }^{2}$ & $63.5 \pm 16.1$ & $72.9 \pm 18.8$ & 0.001 \\
Serum uric acid, mg/dl & $6.9 \pm 1.8$ & $5.9 \pm 1.5$ & 0.001 \\
High-density lipoprotein cholesterol, mg/dl & $43 \pm 13$ & $46 \pm 12$ & 0.307 \\
Low-density lipoprotein cholesterol, mg/dl & $107 \pm 33$ & $114 \pm 33$ & 0.235 \\
Triglyceride, mg/dl & $133 \pm 84$ & $143 \pm 84$ & 0.450 \\
Total serum cholesterol, mg/dl & $176 \pm 43$ & $189 \pm 44$ & 0.070 \\
White blood cell, $10^{3} / \mu l$ & $7.73 \pm 2.73$ & $7.63 \pm 2.47$ & 0.554 \\
Neutrophil/lymphocyte ratio & $3.32 \pm 2.54$ & $3.24 \pm 2.66$ & 0.861 \\
Hemoglobin, g/dl & $13.2 \pm 1.6$ & $13.6 \pm 1.8$ & 0.088 \\
Hematocrit, \% & $40 \pm 4$ & $41 \pm 7$ & 0.104 \\
Red cell distribution width, \% & $15.2 \pm 2.3$ & $15.1 \pm 3.5$ & 0.786 \\
Platelet count, $10^{3} / \mu \mathrm{l}$ & $241 \pm 75$ & $244 \pm 76$ & 0.818 \\
Mean platelet volume, fl & $8.9 \pm 1.1$ & $8.7 \pm 0.9$ & 0.249 \\
\hline
\end{tabular}

Figures in parentheses are percentages.

The baseline demographic characteristics of the study population are shown in table 1 . We found that AF patients with a low peak flow velocity were older $(\mathrm{p}=0.010)$ and female $(\mathrm{p}=0.003)$. We also found that patients with an LAA peak flow velocity $<35 \mathrm{~cm} / \mathrm{s}$ have a decreased eGFR compared with those with an LAA peak flow velocity $\geq 35 \mathrm{~cm} / \mathrm{s}\left(63.5 \pm 16.1\right.$ vs. $72.9 \pm 18.8 \mathrm{ml} / \mathrm{min} / 1.73 \mathrm{~m}^{2}$; $\mathrm{p}=0.001)$. There were no significant differences with regard to other demographic and clinical features or the laboratory findings between the groups.

The LAA peak flow velocity was lower $(24.62 \pm 5.90$ $\mathrm{cm} / \mathrm{s})$ in group 1 compared with group $2(49.28 \pm 13.72$ $\mathrm{cm} / \mathrm{s}$ ), and the difference was statistically significant $(\mathrm{p}=$ 0.001 ). Additionally, the presence of LAA thrombi and SEC was significantly higher in group 1 than in group 2 $(\mathrm{p}=0.027$ and $\mathrm{p}<0.001$, respectively; table 2$)$. Serum acid levels were $6.9 \pm 1.8 \mathrm{mg} / \mathrm{dl}$ for group 1 and $5.9 \pm 1.5 \mathrm{mg} /$ $\mathrm{dl}$ for group 2, and the difference was statistically significant $(\mathrm{p}=0.001)$. Serum uric acid levels were negatively correlated with the LAA blood flow velocity $(r=-0.216$, $\mathrm{p}=0.007)$.

Multivariate regression showed that the serum uric acid level $(\beta=-0.196, \mathrm{p}=0.010)$, age $(\beta=-0.201, \mathrm{p}=$
$0.009)$ and gender differences $(\beta=-0.289, \mathrm{p}<0.001)$ were statistically significant predictors of LAA peak flow velocity (table 3). Although univariate correlation analysis showed diastolic blood pressure, LAA peak flow velocity, SEC, LAA thrombus, left atrial size, left ventricular ejection fraction and GFR were correlated with serum uric acid, and only diastolic blood pressure $(\beta=-0.305, \mathrm{p}=$ $0.016)$ and GFR $(\beta=-0.498, p<0.001)$ were independent predictors of serum uric acid in multivariate regression analysis, using the backward method.

\section{Discussion}

The major finding of this study was that serum uric acid levels were higher in patients with AF and a lower LAA peak flow velocity than in those with AF and a normal LAA peak flow velocity; and, there was an independent association between increased levels of serum uric acid and decreased LAA function in patients with AF. Hence, elevated serum uric acid levels could be considered as a predictor of decreased contractile function of the LAA contributing to the prothrombotic state of patients with AF. 
Table 2. TEE findings of study patients

\begin{tabular}{lccr}
\hline & Group 1 $(\mathrm{n}=87)$ & Group 2 $(\mathrm{n}=66)$ & p values \\
\hline LAA peak flow & & & \\
velocity, cm/s & $24.62 \pm 5.90$ & $49.28 \pm 13.72$ & $<0.001$ \\
LAA thrombus & $19(21.8)$ & $6(9.1)$ & 0.027 \\
SEC & & & $<0.001$ \\
0 & $28(32.2)$ & $51(77.3)$ & \\
$1+$ & $11(12.6)$ & $6(9.1)$ & \\
$2+$ & $19(21.8)$ & $3(4.5)$ & \\
$3+$ & $29(33.3)$ & $6(9.1)$ & \\
\hline
\end{tabular}

Figures in parentheses are percentages.

Table 3. Multivariate regression analysis based on independent variables likely to affect the LAA peak flow velocity

\begin{tabular}{lcrr}
\hline Independent variables & 95\% CI & $\beta$ & $\mathrm{p}^{1}$ \\
\hline Serum uric acid & -3.082 to -0.425 & -0.196 & 0.010 \\
Age & -0.428 to -0.063 & -0.201 & 0.009 \\
Male gender & -14.024 to -4.558 & -0.289 & $<0.001$ \\
Hypertension & -5.910 to 4.088 & -0.029 & 0.719 \\
eGFR & -0.157 to 0.199 & 0.024 & 0.815 \\
LDL-C & -0.014 to 0.128 & 0.119 & 0.115 \\
N/L ratio & -0.771 to 1.170 & 0.033 & 0.685 \\
RDW & -1.207 to 0.453 & -0.068 & 0.371 \\
MPV & -3.154 to 1.300 & -0.062 & 0.412 \\
\hline
\end{tabular}

$\mathrm{CI}=$ Confidence interval; LDL-C = low-density lipoprotein cholesterol; N/L ratio = neutrophil/lymphocyte ratio; $\mathrm{RDW}=$ red cell distribution width; MPV = mean platelet volume.

${ }^{1} \mathrm{p}$ value at the last step, adjusted for the independent variables.

Serum uric acid is the end product of purine metabolism and is produced by the conversion of hypoxanthine to xanthine and then xanthine to uric acid, and both reactions are catalyzed by the XO enzyme [1]. Serum uric acid is a well-established endothelium-injuring factor. Bo et al. [8] showed that elevated serum uric acid levels cause a detrimental effect on endothelial function. However, Ames et al. [9] suggested that serum uric acid has antioxidant effects and may be a powerful scavenger of oxygen and free radicals at physiological concentration levels. These contradictory findings suggest a concentration-dependent dual effect of serum uric acid that exerts beneficial antioxidant properties while within normal limits, whereas harmful pro-oxidant effects could be seen above normal serum concentrations.
Numerous studies have demonstrated that serum uric acid is clearly associated with oxidative stress and inflammation in several pathological conditions. Elevated serum uric acid levels have been suggested as a simple and independent predictor of morbidity and mortality in patients with congestive heart failure [10]. Elevated serum uric acid levels were also found as an independent predictor of cardiovascular events and mortality in patients with coronary artery disease, ST-elevation myocardial infarction [11], hypertension [12], stroke [13], diabetes mellitus [14], metabolic syndrome [15], insulin resistance [14], obesity [16] and renal failure [17]. These findings pointed out that serum uric acid is not only an indicator of the catabolic phase of purine nucleotides but also a manifestation of systemic inflammation in many cardiovascular diseases.

Electrical remodeling is known as the main operative mechanism in paroxysmal AF, whereas atrial structural remodeling holds the principal role in permanent $\mathrm{AF}$ [18]. The consequences of inflammation and oxidative stress can lead to cardiac remodeling and atrial fibrosis as well as increase the susceptibility of AF. However, it is unclear whether serum uric acid participates actively in atrial remodeling or simply represents a marker of the oxidative and inflammatory processes.

Since there is a relationship between serum uric acid levels and markers of inflammation and oxidative stress, the elevated serum uric acid level appears to be an emerging prognostic risk factor of AF. Suzuki et al. [19] reported that the serum uric acid levels were significantly higher in 1,131 AF patients compared with non-AF patients. In another recently published study, Kuwabara et al. [20] showed that serum uric acid levels $\geq 8 \mathrm{mg} / \mathrm{dl}$ might be accepted as an independent predictor of the future development of AF. The association between serum uric acid and AF is largely explained by oxidative stress, which may be responsible for atrial remodeling in patients with AF [21]. XO-induced increased oxidative stress may cause cellular damage and impair the myofibrillar energetics which causes atrial contractile dysfunction [22]. The LA has been found to be more sensitive to oxidative stress [23], and the XO enzyme found in the LA seems to contribute to the increased atrial oxidative stress in patients with AF [21]. Hoeper et al. [2] demonstrated that elevated serum uric acid levels are related to increased right and left atrial filling pressures, which leads to structural and electrophysiological abnormalities that expedite the initiation and continuation of AF. Additionally, Letsas et al. [5] showed a correlation between elevated serum uric acid levels and increased LA diameter as a re- 
sult of the effects of serum uric acid on atrial structural remodeling.

The LAA is a blind-ended pouch, which is embryologically distinct from the main body of the LA. The contractile function of the LAA has been shown to provide a significant contribution to the overall compliance and reservoir function of the LA [7]. Most of the clinical interest in LAA peak flow velocity arises from its relationship with thromboembolism. The contractile function of the LAA and the degree of blood stasis within the LAA have been regarded as substantial determinants of thromboembolic risk in patients with AF. Although much is known about the role of serum uric acid in atrial remodeling, less is known about the potential pathogenetic role of uric acid in maintaining the function of the LAA. In an experimental porcine model of rapid atrial pacing, Dudley et al. [24] demonstrated that increased XO activity was more apparent in the LAA. However, it is unclear whether uric acid participates actively in the mechanical dysfunction of the LAA or simply represents a marker of the oxidative and inflammatory processes.

In the present study, we aimed to investigate the association between LAA function and serum uric acid levels in patients with $\mathrm{AF}$ and observed that uric acid levels were significantly higher in patients with AF and a lower LAA peak flow velocity than in those with $\mathrm{AF}$ and a normal LAA peak flow velocity. Consequently, we suggested that the LAA might be more susceptible to the direct toxic effect of increased serum levels of uric acid, and elevated levels might further impair the myofibrillar energetics of the LAA compared to the other left atrial myocardium.

Additionally, we observed that patients with decreased LAA peak flow velocities were females and older and had a decreased eGFR compared with patients with normal LAA peak flow velocities. It is obvious that atrial fibrosis and susceptibility to AF is increased with aging. It is a matter of controversy in the literature whether there is a gender difference in the incidence of AF. Nevertheless,
Michelena et al. [25] stated that morbidity and mortality are greater in women with $\mathrm{AF}$ than in men with $\mathrm{AF}$. In accordance, we also found that male AF patients generally have normal LAA peak flow velocities compared with females. In multivariate regression analysis, the eGFR did not remain an independent predictor of LAA peak flow velocity.

This study had several limitations. Firstly, the limited number of patients and the retrospective design of the paper create a difficulty for the definite cause-effect relation between serum uric acid and LAA peak flow velocity. Considering that the study was conducted with a special cohort, further studies with larger cohorts are required to improve the generalizability of the results. Secondly, patients were pooled and analyzed irrespective of the duration and type of AF rhythm or a history of heart failure, which could affect the function of the LAA and the level of serum uric acid. Also, we did not evaluate the use of diuretics and statins, which may have a potential effect on the elevated serum uric acid level. Lastly, we did not examine the relative influence of other conventional inflammatory markers.

\section{Conclusions}

In the present study, there was an independent association between high serum uric acid levels and low LAA peak flow velocity. This finding could indicate that a high serum uric acid level seemed to be a predictor of low LAA peak flow velocity in patients with AF. However, further large-scale studies are needed to elucidate the exact pathophysiologic and prognostic role of serum uric acid.

\section{Disclosure Statement}

There are no conflicts of interest.

\section{References}

1 Ruggiero C, Cherubini A, Ble A, et al: Uric acid and inflammatory markers. Eur Heart J 2006;27:1174-1181.

2 Hoeper MM, Hohlfeld JM, Fabel H: Hyperuricaemia in patients with right or left heart failure. Eur Respir J 1999;13:682-685.

-3 Culleton BF, Larson MG, Kannel WB, et al: Serum uric acid and risk for cardiovascular disease and death: the Framingham Heart Study. Ann Intern Med 1999;131:7-13.

Serum Uric Acid Level and LAA Peak Flow Velocity
Korantzopoulos P, Letsas KP, Liu T: Xanthine oxidase and uric acid in atrial fibrillation. Front Physiol 2012;3:150.

5 Letsas KP, Korantzopoulos P, Filippatos GS, et al: Uric acid elevation in atrial fibrillation. Hellenic J Cardiol 2010;51:209-213.

-6 Tamariz L, Agarwal S, Soliman EZ, et al: Association of serum uric acid with incident atrial fibrillation [from the Atherosclerosis Risk in Communities (ARIC) study]. Am J Cardiol 2011;108:1272-1276.
Hoit BD, Shao Y, Tsai LM, et al: Altered left atrial compliance after atrial appendectomy. Influence on left atrial and ventricular filling. Circ Res 1993;72:167-175.

8 Bo S, Gambino R, Durazzo M, et al: Associations between serum uric acid and adipokines, markers of inflammation, and endothelial dysfunction. J Endocrinol Invest 2008; 31:499-504. 
9 Ames BN, Cathcart R, Schwiers E, et al: Uric acid provides an antioxidant defense in humans against oxidant- and radical-caused aging and cancer: a hypothesis. Proc Natl Acad Sci USA 1981;78:6858-6862.

$>10$ Hoieggen A, Alderman MH, Kjeldsen SE, et al: The impact of serum uric acid on cardiovascular outcomes in the LIFE study. Kidney Int 2004;65:1041-1049.

11 Kaya MG, Uyarel H, Akpek M, et al: Prognostic value of uric acid in patients with ST-elevated myocardial infarction undergoing primary coronary intervention. Am J Cardiol 2012;109:486-491.

12 Johnson RJ, Kang DH, Feig D, et al: Is there a pathogenetic role for uric acid in hypertension and cardiovascular and renal disease? Hypertension 2003;41:1183-1190.

13 Taheraghdam AA, Sharifipour E, Pashapour A, et al: Allopurinol as a preventive contrivance after acute ischemic stroke in patients with a high level of serum uric acid: a randomized, controlled trial. Med Princ Pract 2014; 23:134-139.
14 Krishnan E, Pandya BJ, Chung L, et al: Hyperuricemia in young adults and risk of insulin resistance, prediabetes, and diabetes: a 15year follow-up study. Am J Epidemiol 2012; 176:108-116.

15 Al-Meshaweh AF, Jafar Y, Asem M, et al: Determinants of blood uric acid levels in a dyslipidemic Arab population. Med Princ Pract 2012;21:209-216.

16 Horio T, Iwashima Y, Kamide K, et al: Chronic kidney disease as an independent risk factor for new-onset atrial fibrillation in hypertensive patients. J Hypertens 2010;28:1738-1744.

17 Ito H, Abe M, Mifune M, et al: Hyperuricemia is independently associated with coronary heart disease and renal dysfunction in patients with type 2 diabetes mellitus. PLoS One 2011;6:e27817.

18 Nattel S: Therapeutic implications of atrial fibrillation mechanisms: can mechanistic insights be used to improve AF management? Cardiovasc Res 2002;54:347-360.

19 Suzuki S, Sagara K, Otsuka T, et al: Genderspecific relationship between serum uric acid level and atrial fibrillation prevalence. Circ J 2012;76:607-611.
20 Kuwabara M, Niwa K, Niinuma H: Hyperuricemia is an independent risk factor of atrial fibrillation due to electrical remodeling through activation of uric acid transporter (abstract). ACC 2012. J Am Coll Cardiol 2012; 59(suppl A):A163.

21 Korantzopoulos P, Kolettis TM, Galaris D, et al: The role of oxidative stress in the pathogenesis and perpetuation of atrial fibrillation. Int J Cardiol 2007;115:135-143.

22 Mihm MJ, Yu F, Carnes CA, et al: Impaired myofibrillar energetics and oxidative injury during human atrial fibrillation. Circulation 2001;104:174-180.

23 Van Wagoner DR: Molecular basis of atrial fibrillation: a dream or a reality? J Cardiovasc Electrophysiol 2003;14:667-669.

24 Dudley SC Jr, Hoch NE, McCann LA, et al: Atrial fibrillation increases production of superoxide by the left atrium and left atrial appendage: role of the NADPH and xanthine oxidases. Circulation 2005;112:1266-1273.

25 Michelena HI, Powell BD, Brady PA, et al: Gender in atrial fibrillation: ten years later. Gend Med 2010;7:206-217. 